\title{
Frailty Syndrome and the Use of Frailty Indices as a Preoperative Risk Stratification Tool in Spine Surgery: A Review
}

\author{
Trevor Simcox ${ }^{1}$, Derek Antoku $^{1}$, Nickul Jain ${ }^{1}$, Frank Acosta ${ }^{1,2}$, Raymond Hah ${ }^{1,2}$ \\ ${ }^{1}$ Department of Orthopaedic Surgery, Keck School of Medicine of USC, Los Angeles, CA, USA \\ ${ }^{2}$ USC Spine Center at Keck Medicine of USC, Los Angeles, CA, USA
}

\begin{abstract}
This comprehensive narrative literature review aims to extract studies related to frailty indices and their use in elective spine procedures, as limited studies regarding frailty exist in the spine literature. Most studies are retrospective analyses of prospectively collected databases. Evidence suggests a positive correlation between frailty level and mortality rate, postoperative complication rate, length of stay, and the possibility of discharge to a skilled nursing facility; these correlations have been illustrated across various spine procedures. The leading index is the modified frailty index, which measures 11 deficits. The development of more comprehensive frailty indices, such as the Adult Spinal Deformity Frailty Index, are promising and have high predictive value regarding postoperative complication rate in patients with spinal deformity. However, a frailty index that combines clinical, radiographic, and laboratory measures awaits development. Perhaps, the use of a frailty index in preoperative risk stratification for elective spine procedures could serve multiple purposes, including screening for high-risk patients, enhancement of operative decision making, approximation of complication rate for informed decision making, and refinement of perioperative care. Further prospective studies are warranted to determine clinically meaningful interventions in frail individuals.
\end{abstract}

Keywords: Frailty; Adverse events; Elective surgical procedure; Spine; Mortality

\section{Introduction}

Precise prediction of how patients will tolerate elective spine surgery is a significant challenge for spine surgeons. Historically, surgeons have relied on clinical experience, general assessment of overall health, and American Society of Anesthesiologists (ASA) scores to ascertain the ability of patients to tolerate surgery. Limited tools exist to risk stratify patients during preoperative planning objectively. Reportedly, the United States population continues to age, resulting in more patients undergoing surgery at increasingly advanced ages with higher medical comorbidities [1]. Eventually, the demand for a geriatric risk stratification tool will be driven by market forces as healthcare shifts from a fee-for-service to value-based compensation model. In modern healthcare systems, spine surgeons are expected to face pressure to provide systemic value-based outcomes measures for which reimbursement could be fundamentally tied [2,3].

Previously designed tools, such as the ASA Physical Sta-

Received Sep 16, 2018; Revised Jan 30, 2019; Accepted Feb 12, 2019

Corresponding author: Trevor Simcox

Department of Orthopaedic Surgery, NYU Winthrop Hospital, 222 Station Plaza North, Suite 305, Mineola, NY 11501, USA

Tel: +1-516-663-4798, Fax: +1-516-663-2261, E-mail: trevor.simcox@nyumc.org 
tus Classification System, have been useful in evaluating operative risk and estimating perioperative complications. Nevertheless, the ASA scale has poor inter-rater reliability [4-6] and is limited in its capability to precisely risk stratify patients with mild levels of comorbidity $[7,8]$. Recent years have witnessed an increased use of the concept of frailty as a predictor of patients' operative risk. Broadly, frailty is defined as an age-related syndrome characterized by declined physiological reserve across multiple organ systems. To date, several studies have reported frailty syndrome to be an independent risk factor for perioperative complications [9-12], while others have reported in specific populations that high frailty index scores are superior to the ASA in estimating mortality and complication rates $[8,9,13]$. Notably, frailty can be used to help surgeons quantifiably distinguish patients 'physiologic' and chronologic age.

Risk stratification using a frailty index offers a promising tool to identify patients most likely to experience complications to explicate inherent risks of surgery for health professionals, patients, and their families. While several reviews of frailty in surgical patients exist $[10,11,14]$, to the best of our knowledge, this is the first review of frailty related to spine surgery. Hence, this study aims to provide a literature overview as it pertains to the frailty index and elective spine surgery.

\section{Defining Frailty}

Broadly, frailty is defined as an age-related syndrome characterized by reduced physiological reserve across multiple organ systems with a resultant diminished resistance to stressors [15] and a decline in the threshold for decompensation [16]. In addition, frailty could overlap with common geriatric syndromes such as sarcopenia, malnutrition, cachexia, functional disability, and multiple comorbidities $[10,14]$. Frailty syndrome conceptually addresses the distinction between chronological age and physiological age; severely frail patients are not necessarily elderly and not all elderly individuals are frail.

\section{Measuring frailty}

Two major models of defining frailty are the frailty phenotype and the deficit accumulation model, also known as the frailty index. The frailty phenotype model summarizes the multidimensionality of frailty into the following
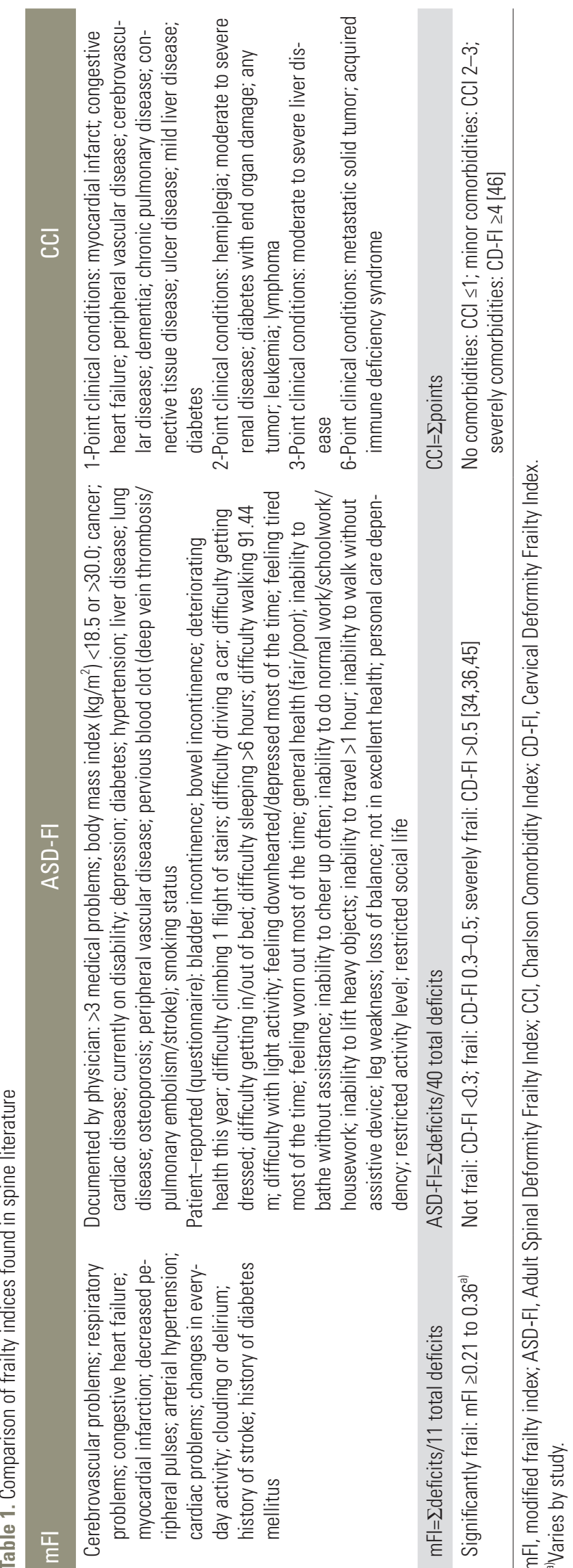
five measures (the Fried Frailty Criteria): unintentional weight loss; grip strength weakness; poor endurance; slow walking speed; and low physical activity; the presence of $\geq 3$ indicates an individual is positive for the frailty phenotype. A study reported these biomarkers as meaningful, as they represent the downward physiologic spiral observed in frailty syndrome [17]. Several studies have proposed using single surrogate measures, such as grip strength or gait speed, as a marker for the frailty phenotype [18-24].

The deficit accumulation model counts the number of deficits in health across multiple organ systems to obtain a single score that is representative of the overall frailty level of patients. Although multiple frailty indices exist, those leading in the spine literature are as follows: modified frailty index (mFI); Charlson Comorbidity Index (CCI); Adult Spinal Deformity Frailty Index (ASD-FI); and Cervical Deformity Frailty Index (CD-FI) [17,25]. Table 1 compares three frailty indices found in the spine literature and lists the deficits measured in each index.

No consensus exists regarding which variables should be used to evaluate the frailty level in spine surgery. While some studies have used the medical history of patients to measure the frailty level, others have used a combination of medical, functional, and laboratory measures to evaluate a frailty score. Given the multifactorial nature of the syndrome, the general consensus is that no single biomarker, taken independently, is adequate for the frailty assessment [15]. Although both frailty index model and frailty phenotype measures have pros and cons, some have inferred that the frailty index model remains the most versatile with wide applicability for both research and clinical use, as it quantifies the concept of frailty $[26,27]$.

\section{Prevalence of frailty}

The prevalence of frailty varies on the basis of the method used to measure it, the study population, and the threshold used to classify an individual as frail. A cohort study of community-dwelling elderly (age, 64-74 years) using the Fried Frailty Criteria reported the overall frailty prevalence to be $8.5 \%$ in females and $4.1 \%$ in males [28]. In the geriatric population undergoing general surgery procedures, studies have reported the frailty prevalence to be as high as $40 \%-50 \%[29,30]$. In the degenerative spine disease (DSD) surgical population, using a threshold of $\mathrm{mFI} \geq 0.27$, the prevalence of clinically significant frailty has been reported to be approximately $4 \%$, with frailty syndrome being 2 times as common in individuals aged $>65$ years [7]. Several frailty studies involving spine procedures reported the percentage of patients with, at least, mild frailty to be $48 \%-60 \%[7,8,31-35]$.

\section{The Use of Frailty Indices in Non-Orthopedic Surgery}

The effect of frailty on surgical outcomes has been investigated in non-orthopedic surgical populations. In addition, studies have shown the application of frailty indices to be useful in estimating postoperative mortality [36], complications [29], increased length of stay (LOS) [29], and discharge to a skilled nursing facility (SNF) [36,37]. Several studies have reported that the use of a frailty index exhibits better predictive value than ASA classification regarding 30-day all-cause postoperative mortality, 1-year all-cause mortality, and risk of nursing facility discharge $[9,13,36]$. Moreover, functional measures of frailty (i.e., ambulation deficits and inability to perform activities of daily living) reportedly predict short-term and mid-term mortality, as well as a multitude of in-hospital morbidities, prolonged LOS, and discharge to SNF, suggesting that preoperative ambulation deficits translate into elevated postoperative risk for pneumonia, re-intubation, prolonged urinary catheterization, and development of urinary tract infection-all of which combined could account for protracted recovery and higher mortality [38].

\section{Frailty and Spine Surgery}

Compared with non-orthopedic literature, few studies regarding frailty indices exist in the spine literature. Most of these studies regarding frailty indices are retrospective analyses of prospectively collected databases, in which a frailty index score is retrospectively evaluated using the preoperative medical history to correlate high frailty index scores with the elevated postoperative complication rate.

The evidence indicates that higher levels of frailty correlate with higher risk of mortality, postoperative complications, prolonged hospital LOS, and more probability of discharge to a rehabilitation facility in both general surgery and, precisely, spine surgical populations. The ability of a frailty index to estimate postoperative complications varies on the basis of the study population, invasiveness of the procedure, and index used to measure frailty. Table 2 summarizes pertinent studies in the spine literature, categorizing each study 


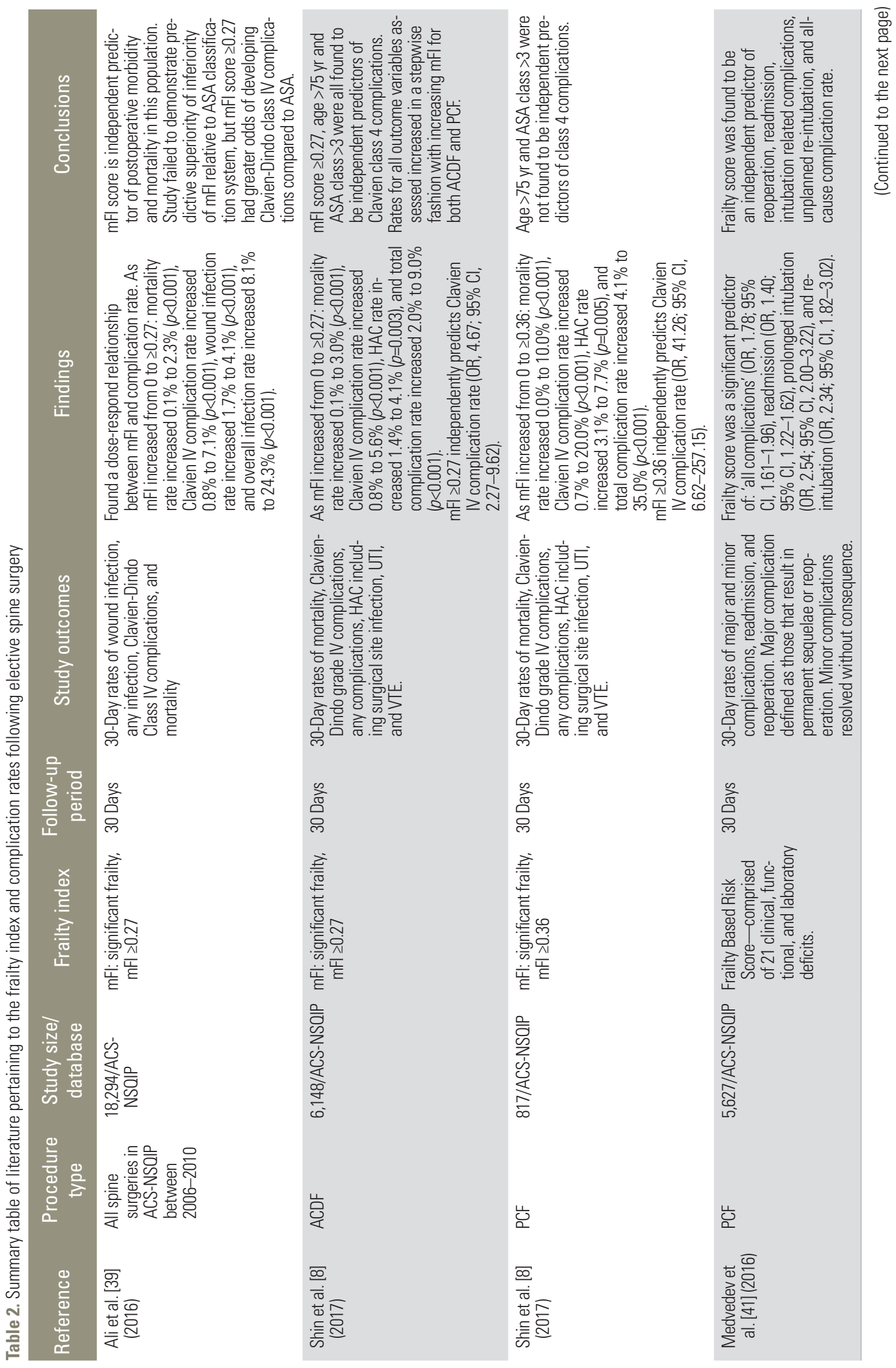




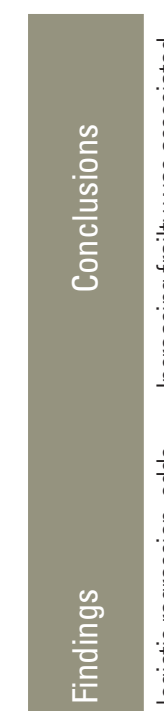

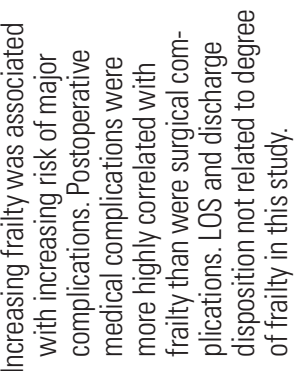

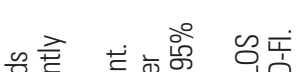

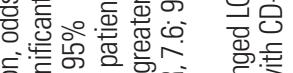

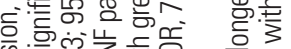

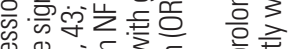

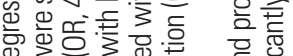

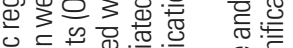

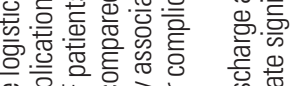

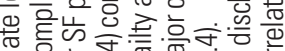
薃

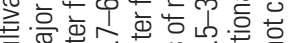

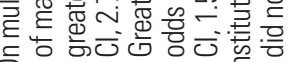

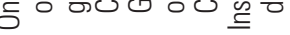

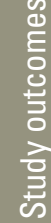

을은

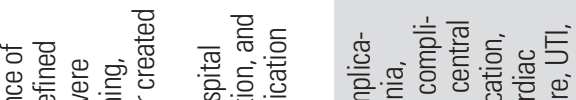

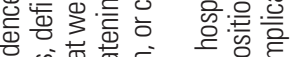

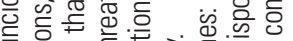

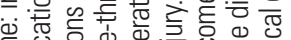

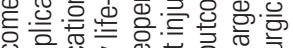

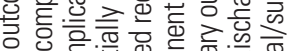

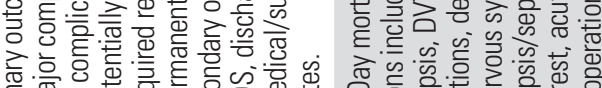

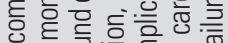

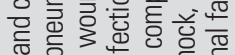

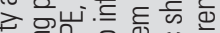

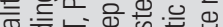

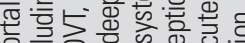

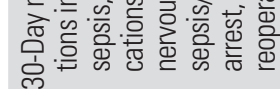

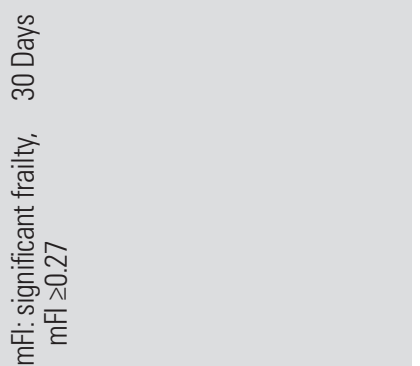

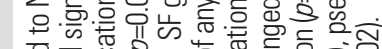

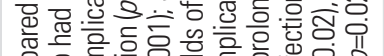

c

客

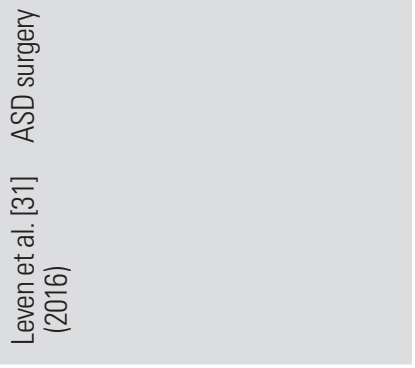

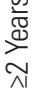

ஸें

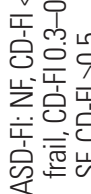

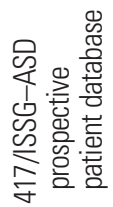

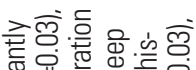

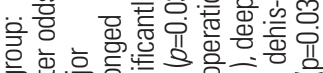

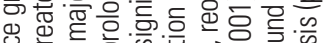

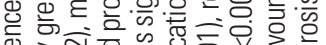

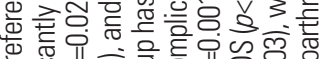

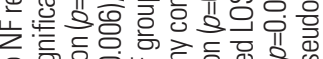

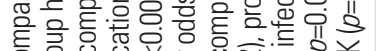

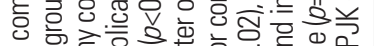

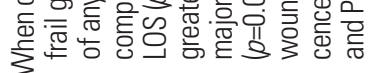

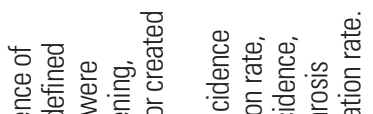

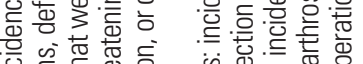

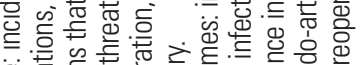

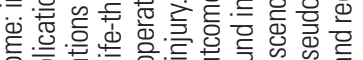

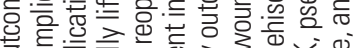
亏

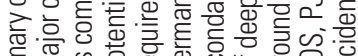

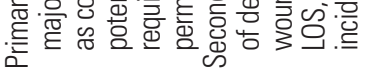

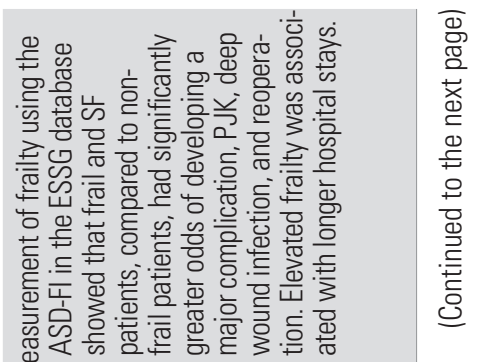

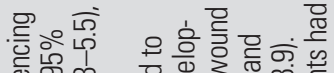
竹

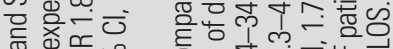

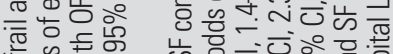
w

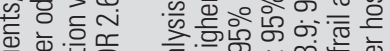

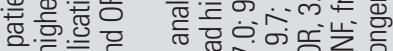

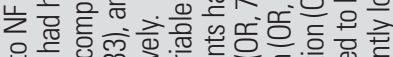

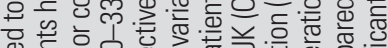

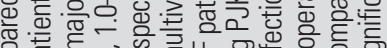
हूँ त

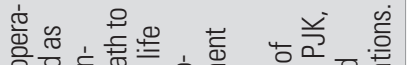

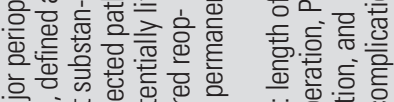

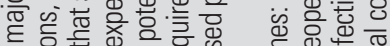
बं

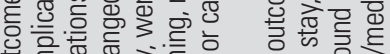

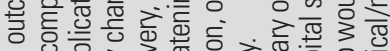
. ㄴ.ᅳ

$\frac{\sqrt{\pi}}{\stackrel{\infty}{\infty}}$

సิ

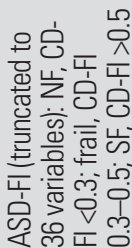

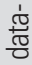

曲

这苋

zo

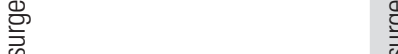

年

ल ल

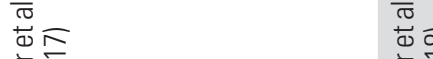

高定 


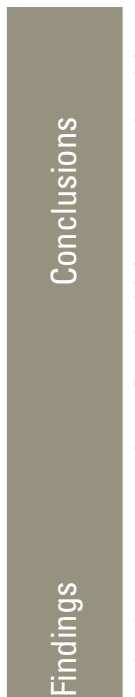

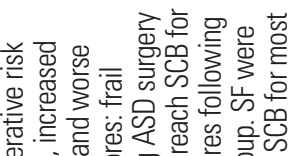

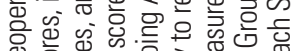

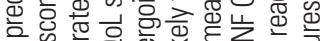

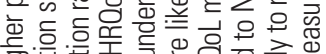
을 떵

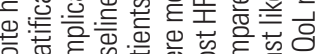

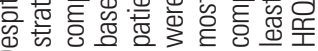

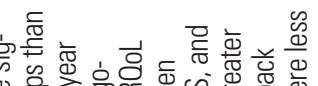

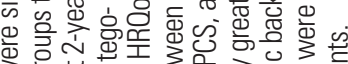
了证丧芯.

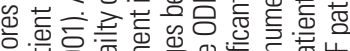

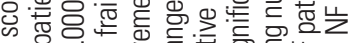
产

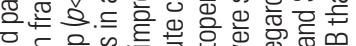

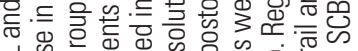

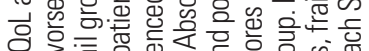

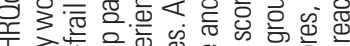

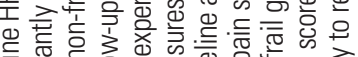

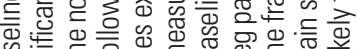
赵

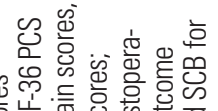

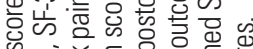
넝 总言

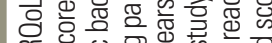

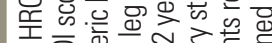

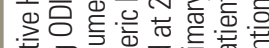

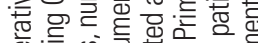

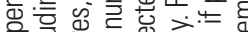

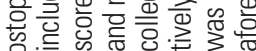

\section{윽 을} 。

$\underset{N}{\stackrel{\infty}{\mathbb{d}}}$

ஸ்

ब

प्र

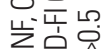

章传

安密它

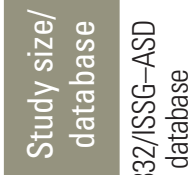

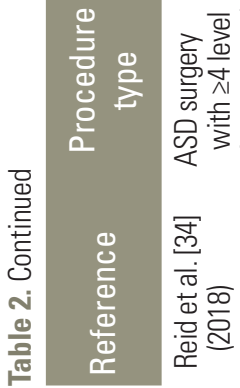

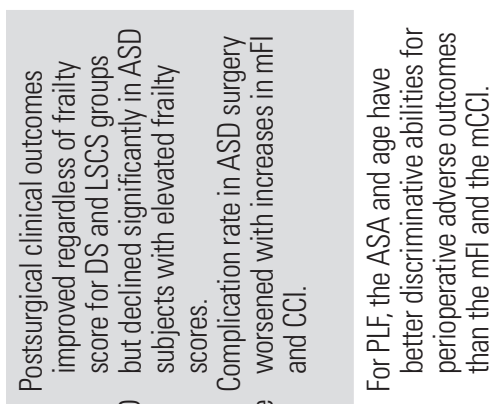

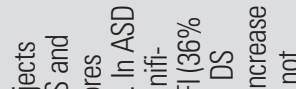

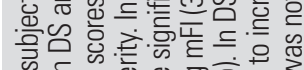

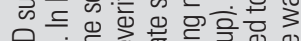

足倇

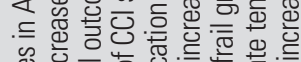

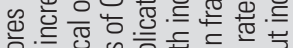

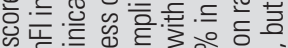

站

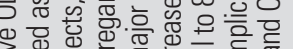

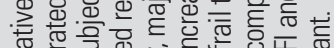

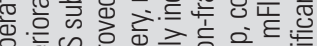

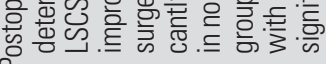

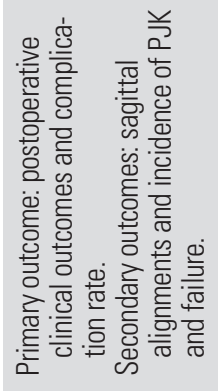

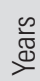

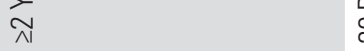

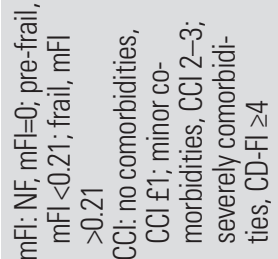

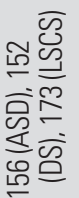

क्षेट्र

흔흥

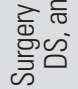

寻

त्त

휴므

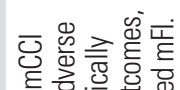
记

要需专

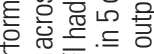

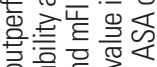

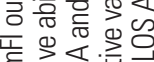

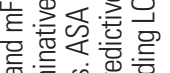

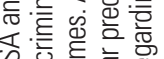

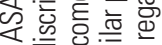

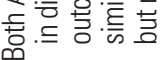

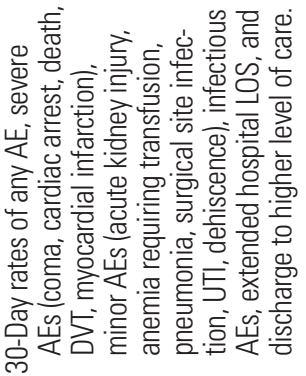

商

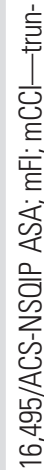

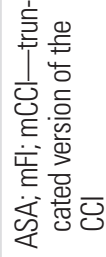

苮

產

○

롬

लु

兽

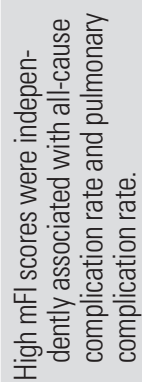

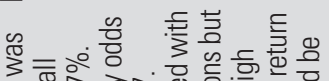

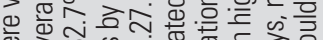
ब्र 등 성 \& 잉을

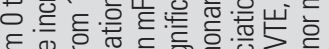
등. 은

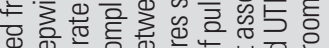

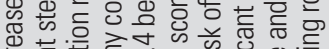

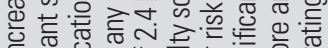

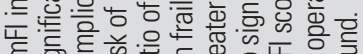

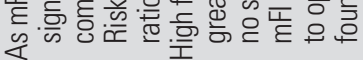

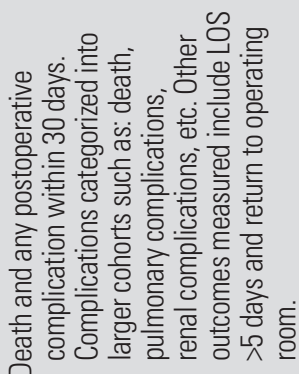

هั

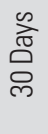

एप

言

岁

㐫

है를

흥ㅎㅁ

产密. 흔

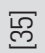

लं

芯令 


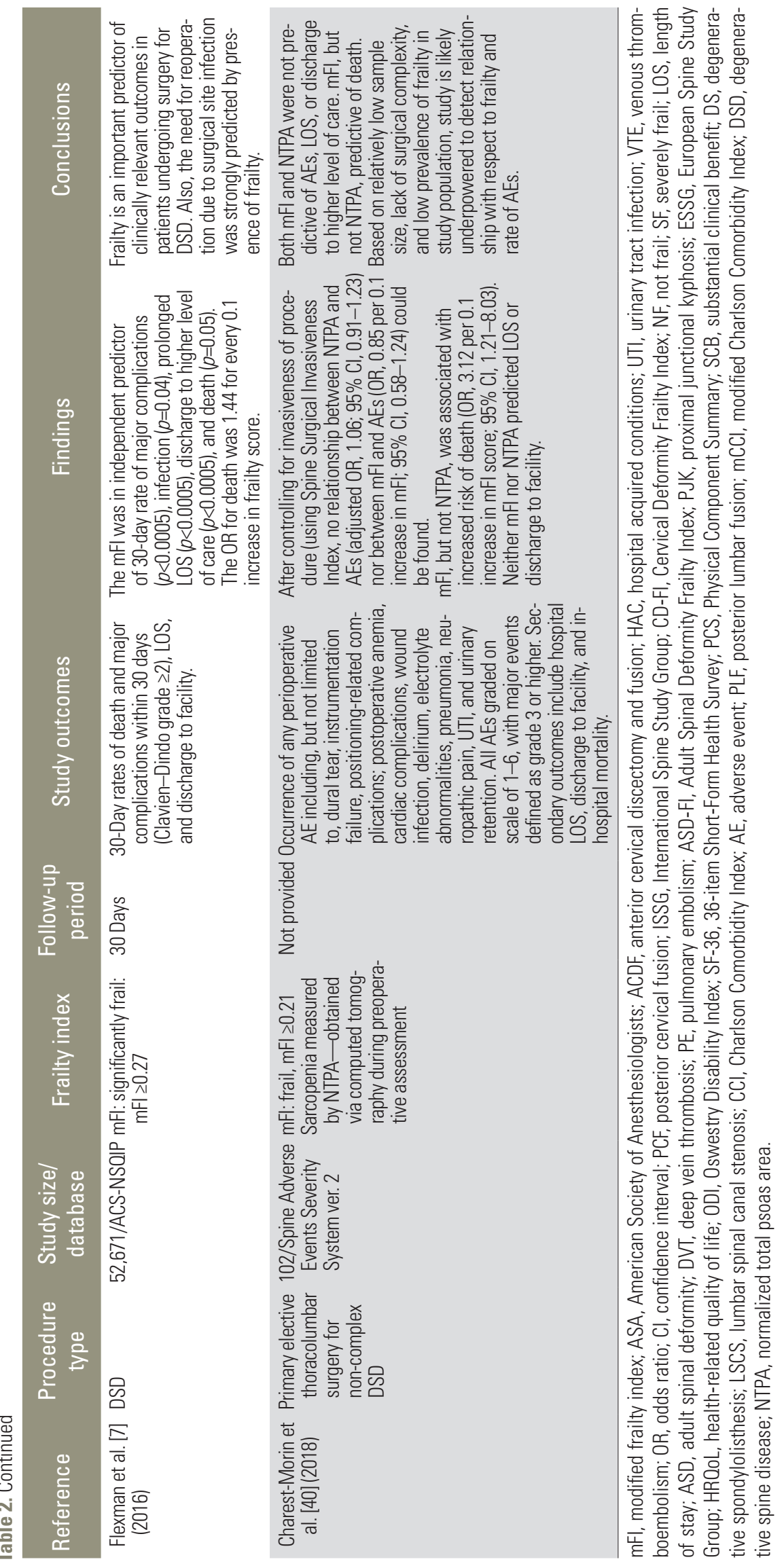


by the procedure type, and discusses the predictive capacity of the frailty index as it relates to postoperative complications associated with that specific procedure.

\section{Postoperative mortality}

Multiple studies have reported that increased frailty index scores correlate with postoperative mortality. From the ACS-NSQIP database, increasing $\mathrm{mFI}$ scores were found to be an independent predictor of 30-day mortality in the general spine surgery population [39], as well as in patients undergoing anterior cervical discectomy and fusion (ACDF) [8], posterior cervical fusion (PCF) [8], adult spinal deformity (ASD) procedures [31], and procedures for degenerative spine conditions [7]. Charest-Morin et al. [40] reported that the mFI was superior to the presence of sarcopenia in estimating mortality in 102 patients undergoing primary elective surgery for noncomplex DSD. Nevertheless, increased mFI scores did not correlate with increased 30-day mortality rates for patients undergoing anterior lumbar interbody fusion (ALIF) in one study [35].

\section{Postoperative complications}

Across various spine procedures, increasing frailty index scores correlated with higher rates of all-cause complications. In the ACS-NSQIP dataset, Ali et al. [39] reported a positive correlation between the $\mathrm{mFI}$ and the 30-day complication rate in the general spine surgical population; this correlation between the increasing frailty score and the 30-day all-cause complication rate has also been reported in patients undergoing ACDF [8], PCF [8,41], ALIF [35], and ASD surgery [31].

The preoperative stratification of patients into tiered risk categories using a frailty index score could offer a surgeon with a predictive tool for major life-threatening complications; this has been reported in the general spine surgery population [39], as well as in patients undergoing cervical spinal deformity surgery [42] and ASD surgery $[32,43,44]$. In these studies, individuals were assigned to tiered risk groups based on frailty index threshold values; assignment to a high-risk group was predictive of the postoperative complication rate.

Some studies reported that frailty syndrome correlated with an elevated risk of infection $[7,32,39,43]$ and pulmonary complications $[35,41]$. Ali et al. [39] reported that in increasing frailty levels markedly elevated both wound infection rate and total postoperative infection rate in the general spine surgery population. Medvedev et al. [41], using a frailty-based risk score comprising of 20 items, reported that frailty index score was an independent predictor of unplanned re-intubation and elevated intubation-related complication rates. In ACS-NSQIP patients undergoing ALIF, Phan et al. [35] reported that elevated mFI correlated with a higher risk of pulmonary complications but not wound complications. These findings corroborated that of non-orthopedic frailty studies that demonstrate how frailty syndrome and deficits in preoperative mobility could translate into increased perioperative pulmonary and infection risk [38].

\section{Reoperation rate}

Frailty syndrome independently correlates with the reoperation rate in patients undergoing surgery for DSD [7], ASD [31,32,43], and PCF [41], while a study of patients undergoing ALIF failed to establish a marked correlation between the frailty score and the reoperation rate. In patients undergoing surgery for ASD, Leven et al. [31] reported that mFI scores of 0.09 compared with 0.18 exhibited a higher predictive value for reoperation than age $>60$ years and obesity class $>$ III (body mass index $>40 \mathrm{~kg} /$ $\mathrm{m}^{2}$ ). In DSD surgery, Flexman et al. [7] reported that the need for reoperation because of surgical site infection was robustly estimated by the presence of frailty.

\section{Prolonged length of stay, institutional discharge, and readmission}

To date, multiple studies of non-orthopedic surgeries have demonstrated a correlation of frailty syndrome with prolonged LOS and elevated risk of institutional discharge $[13,29,36-38,45]$. In the spine literature, the data are mixed, with conflicting data $[7,32,35,40,42,43]$ on the correlation between frailty syndrome and prolonged LOS or institutional discharge.

Regarding readmission, high frailty-based risk scores correlated with increased 30-day readmission rates in patients undergoing PCF [41]. In ACDF, Phan et al. [46] reported a significant and independent correlation between ASA class 4, cardiac comorbidity, and prior stroke and 30-day rate of hospital admissions; considering several of these factors also correlated with high levels of frailty, future studies investigating readmission and the frailty 
index could yield similar results.

\section{Quality of life in patients with adult spinal deformity}

In the ASD literature, mixed results exist regarding whether frailty is useful in estimating the odds of functional improvement. A study of patients who underwent ASD surgery reported that the proportion of moderately frail patients to reach substantial clinical benefit (SCB) at the 2-year follow-up was higher than that of non-frail patients regarding several health-related quality of life measures, including the Oswestry Disability Index (ODI), the 36-item Short-Form Health Survey Physical Compo- nent Summary score, and numeric leg pain. Reportedly, severely frail patients were least likely to reach SCB [34]. Another study of frailty in ASD surgery did not find this correlation; rather the postsurgical ODI scores declined markedly as frailty and comorbidity level increased [44].

\section{Discussion}

In the surgical community, the concept of frailty and the use of the frailty index has been gradually gaining acceptance; it is imperative that spine surgeons recognize the correlation between frailty and perioperative risk in the geriatric population. Overall, the literature indicates that
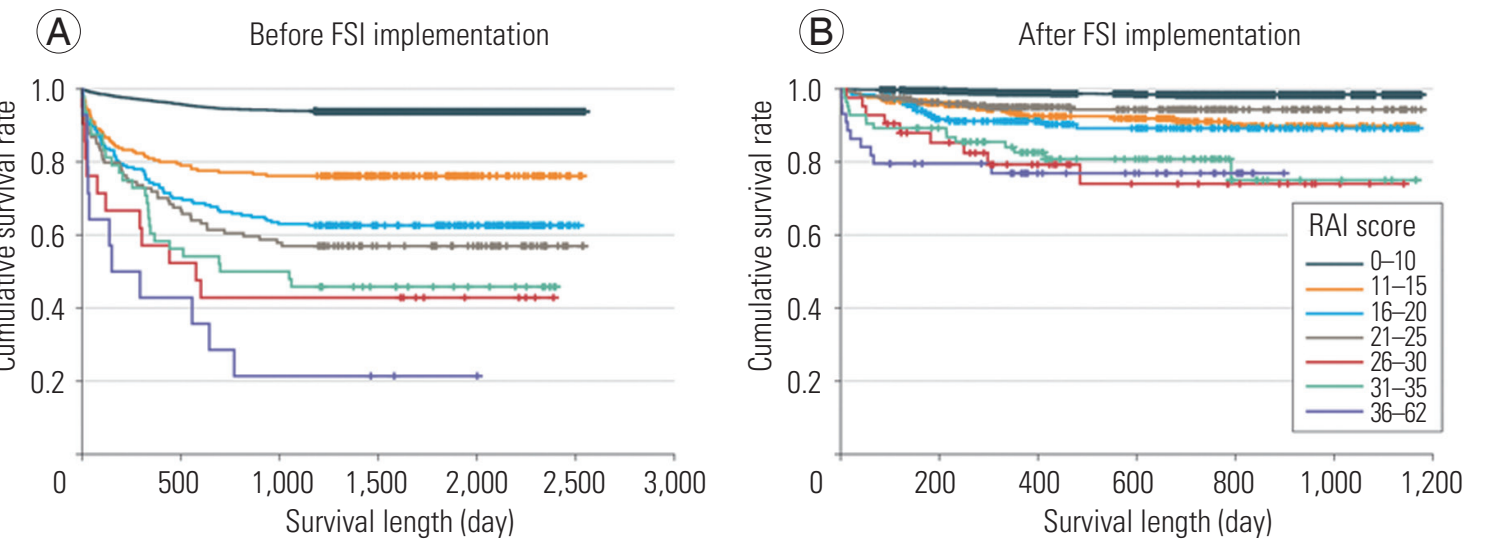

\begin{tabular}{|c|c|c|c|c|c|c|c|}
\hline \multicolumn{8}{|c|}{$\begin{array}{l}\text { No. at risk } \\
\text { RAl score }\end{array}$} \\
\hline 0-10 & 4,654 & 4,459 & 4,377 & 3,313 & 1,537 & 142 & 0 \\
\hline $11-15$ & 210 & 166 & 160 & 107 & 40 & 3 & 0 \\
\hline 16-20 & 214 & 150 & 135 & 105 & 75 & 1 & 0 \\
\hline $21-25$ & 114 & 76 & 66 & 45 & 33 & 3 & 0 \\
\hline $26-30$ & 21 & 11 & 9 & 9 & 4 & 0 & 0 \\
\hline $31-35$ & 48 & 27 & 24 & 15 & 9 & 0 & 0 \\
\hline $36-62$ & 14 & 6 & 3 & 2 & 1 & 0 & 0 \\
\hline
\end{tabular}

\begin{tabular}{|c|c|c|c|c|c|c|c|}
\hline $\begin{array}{l}\text { No. at risk } \\
\text { RAl score }\end{array}$ & & & & & & & \\
\hline 0-10 & 2,922 & 2,560 & 2,027 & 1,707 & 1,178 & 506 & 0 \\
\hline $11-15$ & 340 & 261 & 176 & 139 & 64 & 27 & 0 \\
\hline $16-20$ & 192 & 153 & 110 & 83 & 53 & 26 & 0 \\
\hline $21-25$ & 254 & 224 & 155 & 125 & 49 & 17 & 0 \\
\hline $26-30$ & 42 & 32 & 20 & 13 & 9 & 3 & 0 \\
\hline $31-35$ & 84 & 71 & 49 & 35 & 11 & 5 & 0 \\
\hline $36-62$ & 44 & 31 & 22 & 18 & 8 & 0 & 0 \\
\hline
\end{tabular}

Fig. 1. The implementation of a FSI at a single medical center resulted in significant improvement in postoperative survival among frail patients. The Kaplan-Meier survival curves of cohorts before (A) and after (B) the FSI implementation. Individuals are stratified into cohorts based on the RAI, a 14-item frailty index. Stratification demonstrates that survival benefit was highest in individuals with the highest levels of frailty. The sample included all 9,153 patients (5,275 before FSI implementation and 3,878 after FSI implementation). Mantel-Cox log rank tests for differences in the survival distribution are as follows ( $p<0.001$ for overall difference before and after FSI implementation). Before FSI implementation, the lowest 2 strata of frailty were different from each other and from all the other strata (all $p<0.001$ ). There was no difference between the 16 to 20 and 21 to 25 RAl strata ( $p=0.31$ ), although the 16 to 20 RAl stratum was different from the highest 3 strata of frailty (all $p<0.05$ ). The 21 to 25 RAl stratum was not different from the 26 to $30(p=0.16)$ or the 31 to $35(p=0.24)$ RAl stratum, but it was different from the 36 to 62 RAl stratum (p=0.004). Although the lines of the highest 3 strata diverge, the differences did not reach statistical significance (all $p>0.05$ ); however, this is likely attributable to the low numbers in these RAI strata. After FSI implementation, the lowest frailty stratum was different from all others $(p<0.001)$, but there was no difference between the next RAI strata (e.g., 11-15, 16-20, and 21-25; all $p>0.20$ ), although these 3 were different from the top 3 strata (all $p<0.03$ ). There was no difference between the top 3 strata (e.g., 26-30, 31-35, and 36-62; all $p>0.50$ ), but they were all different from each of the lowest 3 strata (all $p<0.05$ ). Hash marks indicate censored data. FSI, Frailty Screening Initiative; RAI, Risk Analysis Index. Reprinted from Hall et al. JAMA Surg 2017;152:233-40, with permission of American Medical Association [47]. 
increasing levels of frailty, as measured by a frailty index, independently predict the postoperative mortality rate, complication rate, reoperation rate, prolonged LOS, and readmission rate.

Perhaps, a spine-specific frailty index could be a useful objective measure that could serve multiple purposes, including preoperative screening for high-risk patients and estimation of the complication rate for use in multidisciplinary conferences, especially for high-risk ASD patients. Reportedly, preoperative screening using a frailty index, followed by a multidisciplinary review of operative decision making, markedly improves postoperative mortality in elective surgery. Hall et al. [47] reported that the institution of a Frailty Screening Initiative (FSI) in patients undergoing elective surgery led to marked mortality benefit among significantly frail patients, with 30-day, 6-month, and 1-year mortality rates in frail patients falling from $12.2 \%$ to $3.8 \%, 23.9 \%$ to $7.7 \%$, and $34.5 \%$ to $11.7 \%$, respectively. Fig. 1 presents their Kaplan-Meier survival curve before and after the FSI implementation [47]. In the spine population, elevated frailty index scores have been reported as an independent predictor of surgical complications. Preoperative screening using a frailty index might identify high-risk patients, who subsequently qualify for case discussion in a multidisciplinary conference.

In complex ASD surgeries, the implementation of risk reduction protocols, such as the Seattle Spine Team Protocol, have accounted for decreased complication rates $[48,49]$. Sethi et al. [49] reported that the combined use of a multidisciplinary spinal surgery conference, a patient education course, dual operating surgeons, a dedicated complex spine anesthesia team, and enhanced intraoperative monitoring of laboratory measurements and vitals, led to a $51 \%$ decline in the 30 -day complication rate for complex ASD surgery patients. The use of frailty index scores and the consequent estimation of mortality and complication rate could provide clinically pertinent information to the multidisciplinary team. In addition, objective risk stratification scores, such as the Seattle Spine Score for ASD surgery, have exhibited superiority in predictive capacity regarding the 30-day complication rate compared with an expert physician using medical history alone [50]. The frailty index is a conceptually similar model for objectively measuring risk and might benefit spine surgeons in the context of screening for high-risk geriatric patients, enhancing operative decision making, and refining postoperative care.
The spine literature offers limited information on the implementation of a frailty index. To the best of our knowledge, no prospective studies exist regarding frailty and spine surgery [42]. Without prospective data, we are limited in our ability to assess the impact of a frailty diagnosis on operative decisions and perioperative care. In addition, the ACS-NSQIP database studies are limited by 30-day follow-up and might not capture the level of surgical complexity. In ASD surgery patients, controlled for the complexity of the procedure, Miller et al. [32] reported an independent correlation between frailty and complication rate. However, Charest-Morin et al. [40] failed to demonstrate this correlation in DSD surgery.

The current body of literature predominantly uses the mFI, although recent studies have adopted alternative indices such as the CCI, CD-FI, or ASD-FI $[32,34,42,44]$. The mFI score evaluation is convenient from medical history, but indices that account for a higher number of variables and comprise relevant laboratory or functional measures have enhanced accuracy in measuring the frailty level. No consensus exists in the spine literature regarding which particular frailty index is optimal for risk stratification. Perhaps, a frailty index that combines clinical and medical history information, comorbidities, objective laboratory values, and radiographic parameters, such as the bone density, could be the most robust, predictive, accurate, and useful for spine surgeons.

Specialty-specific indices, such as the Metastatic Spinal Tumor Frailty Index, could predict postoperative outcomes with higher accuracy because of only selecting variables with the highest correlation to poor outcomes. Perhaps, the development of a spine-specific frailty index, which involves radiographic measures and/or relevant laboratory measures, might have improved the correlation between the index score and the complication rate.

\section{Conclusions}

In conclusion, currently available frailty indices are adequate in predicting the perioperative complication risk and could be useful in the preoperative screening of geriatric spine patients and guiding surgical management.

\section{Conflict of Interest}

No potential conflict of interest relevant to this article was reported. 


\section{ORCID}

Trevor Simcox: https://orcid.org/0000-0001-6435-5407

Derek Antoku: https://orcid.org/000-0002-5293-5233

Frank Acosta: https://orcid.org/0000-0002-8352-199X

Raymond Hah: https://orcid.org/0000-0001-7513-3519

\section{References}

1. Vincent GK, Velkoff VA. The next four decades: the older population in the United States: 2010 to 2050. Washington (DC): US Department of Commerce, Economics and Statistics Administration, US Census Bureau; 2010.

2. Porter ME. Value-based health care delivery. Ann Surg 2008;248:503-9.

3. Porter ME. A strategy for health care reform: toward a value-based system. N Engl J Med 2009;361:109-12.

4. Owens WD, Felts JA, Spitznagel EL Jr. ASA physical status classifications: a study of consistency of ratings. Anesthesiology 1978;49:239-43.

5. Haynes SR, Lawler PG. An assessment of the consistency of ASA physical status classification allocation. Anaesthesia 1995;50:195-9.

6. Mak PH, Campbell RC, Irwin MG; American Society of Anesthesiologists. The ASA physical status classification: inter-observer consistency. American Society of Anesthesiologists. Anaesth Intensive Care 2002;30:633-40.

7. Flexman AM, Charest-Morin R, Stobart L, Street J, Ryerson CJ. Frailty and postoperative outcomes in patients undergoing surgery for degenerative spine disease. Spine J 2016;16:1315-23.

8. Shin JI, Kothari P, Phan K, et al. Frailty index as a predictor of adverse postoperative outcomes in patients undergoing cervical spinal fusion. Spine (Phila Pa 1976) 2017;42:304-10.

9. Farhat JS, Velanovich V, Falvo AJ, et al. Are the frail destined to fail? Frailty index as predictor of surgical morbidity and mortality in the elderly. J Trauma Acute Care Surg 2012;72:1526-30.

10. Robinson TN, Walston JD, Brummel NE, et al. Frailty for surgeons: review of a national institute on aging conference on frailty for specialists. J Am Coll Surg 2015;221:1083-92.

11. Buigues C, Juarros-Folgado P, Fernandez-Garrido J, Navarro-Martinez R, Cauli O. Frailty syndrome and pre-operative risk evaluation: a systematic review. Arch Gerontol Geriatr 2015;61:309-21.

12. Reisinger KW, van Vugt JL, Tegels JJ, et al. Functional compromise reflected by sarcopenia, frailty, and nutritional depletion predicts adverse postoperative outcome after colorectal cancer surgery. Ann Surg 2015;261:345-52.

13. Kim SW, Han HS, Jung HW, et al. Multidimensional frailty score for the prediction of postoperative mortality risk. JAMA Surg 2014;149:633-40.

14. Partridge JS, Harari D, Dhesi JK. Frailty in the older surgical patient: a review. Age Ageing 2012;41:142-7.

15. Rodriguez-Manas L, Feart C, Mann G, et al. Searching for an operational definition of frailty: a Delphi method based consensus statement: the frailty operative definition-consensus conference project. J Gerontol A Biol Sci Med Sci 2013;68:62-7.

16. Bagshaw SM, McDermid RC. The role of frailty in outcomes from critical illness. Curr Opin Crit Care 2013;19:496-503.

17. Fried LP, Tangen CM, Walston J, et al. Frailty in older adults: evidence for a phenotype. J Gerontol A Biol Sci Med Sci 2001;56:M146-56.

18. Afilalo J, Eisenberg MJ, Morin JF, et al. Gait speed as an incremental predictor of mortality and major morbidity in elderly patients undergoing cardiac surgery. J Am Coll Cardiol 2010;56:1668-76.

19. Guo CB, Zhang W, Ma DQ, Zhang KH, Huang JQ. Hand grip strength: an indicator of nutritional state and the mix of postoperative complications in patients with oral and maxillofacial cancers. Br J Oral Maxillofac Surg 1996;34:325-7.

20. Robinson TN, Wu DS, Sauaia A, et al. Slower walking speed forecasts increased postoperative morbidity and 1-year mortality across surgical specialties. Ann Surg 2013;258:582-8.

21. Savva GM, Donoghue OA, Horgan F, O'Regan C, Cronin H, Kenny RA. Using timed up-and-go to identify frail members of the older population. J Gerontol A Biol Sci Med Sci 2013;68:441-6.

22. Syddall H, Cooper C, Martin F, Briggs R, Aihie Sayer A. Is grip strength a useful single marker of frailty? Age Ageing 2003;32:650-6.

23. Abellan van Kan G, Rolland Y, Bergman H, Morley JE, Kritchevsky SB, Vellas B. The I.A.N.A Task Force on frailty assessment of older people in clinical practice. J Nutr Health Aging 2008;12:29-37. 
24. Vermeulen J, Neyens JC, van Rossum E, Spreeuwenberg MD, de Witte LP. Predicting ADL disability in community-dwelling elderly people using physical frailty indicators: a systematic review. BMC Geriatr 2011;11:33.

25. Velanovich V, Antoine H, Swartz A, Peters D, Rubinfeld I. Accumulating deficits model of frailty and postoperative mortality and morbidity: its application to a national database. J Surg Res 2013;183:10410 .

26. de Vries NM, Staal JB, van Ravensberg CD, Hobbelen JS, Olde Rikkert MG, Nijhuis-van der Sanden MW. Outcome instruments to measure frailty: a systematic review. Ageing Res Rev 2011;10:104-14.

27. Song X, Mitnitski A, Rockwood K. Prevalence and 10 -year outcomes of frailty in older adults in relation to deficit accumulation. J Am Geriatr Soc 2010;58:681-7.

28. Syddall H, Roberts HC, Evandrou M, Cooper C, Bergman H, Aihie Sayer A. Prevalence and correlates of frailty among community-dwelling older men and women: findings from the Hertfordshire Cohort Study. Age Ageing 2010;39:197-203.

29. Makary MA, Segev DL, Pronovost PJ, et al. Frailty as a predictor of surgical outcomes in older patients. J Am Coll Surg 2010;210:901-8.

30. Sundermann S, Dademasch A, Praetorius J, et al. Comprehensive assessment of frailty for elderly highrisk patients undergoing cardiac surgery. Eur J Cardiothorac Surg 2011;39:33-7.

31. Leven DM, Lee NJ, Kothari P, et al. Frailty index is a significant predictor of complications and mortality after surgery for adult spinal deformity. Spine (Phila Pa 1976) 2016;41:E1394-401.

32. Miller EK, Neuman BJ, Jain A, et al. An assessment of frailty as a tool for risk stratification in adult spinal deformity surgery. Neurosurg Focus 2017;43:E3.

33. Ondeck NT, Bohl DD, Bovonratwet P, et al. Discriminative ability of commonly used indices to predict adverse outcomes after poster lumbar fusion: a comparison of demographics, ASA, the modified Charlson Comorbidity Index, and the modified Frailty Index. Spine J 2018;18:44-52.

34. Reid DB, Daniels AH, Ailon T, et al. Frailty and health-related quality of life improvement following adult spinal deformity surgery. World Neurosurg 2018;112:e548-54.
35. Phan K, Kim JS, Lee NJ, et al. Frailty is associated with morbidity in adults undergoing elective anterior lumbar interbody fusion (ALIF) surgery. Spine J 2017;17:538-44.

36. Robinson TN, Eiseman B, Wallace JI, et al. Redefining geriatric preoperative assessment using frailty, disability and co-morbidity. Ann Surg 2009;250:44955.

37. Robinson TN, Wallace JI, Wu DS, et al. Accumulated frailty characteristics predict postoperative discharge institutionalization in the geriatric patient. J Am Coll Surg 2011;213:37-42.

38. Lee DH, Buth KJ, Martin BJ, Yip AM, Hirsch GM. Frail patients are at increased risk for mortality and prolonged institutional care after cardiac surgery. Circulation 2010;121:973-8.

39. Ali R, Schwalb JM, Nerenz DR, Antoine HJ, Rubinfeld I. Use of the modified frailty index to predict 30day morbidity and mortality from spine surgery. J Neurosurg Spine 2016;25:537-41.

40. Charest-Morin R, Street J, Zhang H, et al. Frailty and sarcopenia do not predict adverse events in an elderly population undergoing non-complex primary elective surgery for degenerative conditions of the lumbar spine. Spine J 2018;18:245-54.

41. Medvedev G, Wang C, Cyriac M, Amdur R, O’Brien J. Complications, readmissions, and reoperations in posterior cervical fusion. Spine (Phila $\mathrm{Pa} 1976$ ) 2016;41:1477-83.

42. Miller EK, Ailon T, Neuman BJ, et al. Assessment of a novel adult cervical deformity frailty index as a component of preoperative risk stratification. World Neurosurg 2018;109:e800-6.

43. Miller EK, Vila-Casademunt A, Neuman BJ, et al. External validation of the adult spinal deformity (ASD) frailty index (ASD-FI). Eur Spine J 2018;27:2331-8.

44. Yagi M, Fujita N, Okada E, et al. Impact of frailty and comorbidities on surgical outcomes and complications in adult spinal disorders. Spine (Phila Pa 1976) 2018;43:1259-67.

45. Dasgupta M, Rolfson DB, Stolee P, Borrie MJ, Speechley M. Frailty is associated with postoperative complications in older adults with medical problems. Arch Gerontol Geriatr 2009;48:78-83.

46. Phan K, Kim JS, Lee NJ, Kothari P, Cho SK. Relationship between ASA scores and 30-day readmissions in patients undergoing anterior cervical discectomy and 
fusion. Spine (Phila Pa 1976) 2017;42:85-91.

47. Hall DE, Arya S, Schmid KK, et al. Association of a frailty screening initiative with postoperative survival at 30, 180, and 365 days. JAMA Surg 2017;152:23340.

48. Sethi RK, Pong RP, Leveque JC, Dean TC, Olivar SJ, Rupp SM. The Seattle Spine Team approach to adult deformity surgery: a systems-based approach to perioperative care and subsequent reduction in perioperative complication rates. Spine Deform 2014;2:95103.
49. Sethi R, Buchlak QD, Yanamadala V, et al. A systematic multidisciplinary initiative for reducing the risk of complications in adult scoliosis surgery. J Neurosurg Spine 2017;26:744-50.

50. Buchlak QD, Yanamadala V, Leveque JC, Edwards A, Nold K, Sethi R. The Seattle spine score: predicting 30 -day complication risk in adult spinal deformity surgery. J Clin Neurosci 2017;43:247-55. 